\title{
CHARACTERS AND GENEROSITY OF PERMUTATION GROUPS
}

\author{
JAN SAXL
}

ABSTRACT. A necessary and sufficient character condition is obtained for a group $G$ to be generously $k$-fold transitive. This is similar to an old theorem of $F$ robenius on multiply transitive groups.

Let $G$ be a permutation group on a set $\Omega$ of $n$ points. Let $\chi^{\lambda}$ be the irreducible character of the symmetric group $S_{n}$ corresponding to the partition $\lambda$ of $n$. Let $k, r$ and $s$ be nonnegative integers such that $k=r+s$. It is a well-known fact, due essentially to Frobenius, that $G$ is $k$-fold transitive on $\Omega$ if. and only if $\left(\chi^{\lambda}, \chi^{\mu}\right)_{G}=\delta_{\lambda \mu}$ for any characters $\chi^{\lambda}, \chi^{\mu}$ of $s_{n}$ with dimension $\chi^{\lambda} \leq r$, dimension $\chi^{\mu} \leq s$. Here $\delta_{\lambda \mu}$ is equal to 1 if $\lambda=\mu$ and equals 0 otherwise; and the dimension of the character $\chi^{\lambda}$ corresponding to the partition $\lambda=\left(\lambda_{1}, \cdots, \lambda_{i}\right)$ is defined to be $n-\lambda_{1}$ (see Lemma 2 (b) for an alternative definition). For a proof of this result see [5, 14.3].

Following P. M. Neumann we say that $G$ is generously $k$-fold transitive if for any $\Delta \subseteq \Omega$ with $|\Delta|=k+1$, the setwise stabilizer $G_{\Delta}$ acts as the symmetric group $S_{k+1}$ on $\Delta$. The aim of this note is to give a necessary and sufficient condition similar to the one above for a $k$-fold transitive group to be generously $k$-fold transitive.

Theorem. Let $G$ be $k \cdot f o l d$ transitive on a set $\Omega$ of $n$ points, $n>2 k$. Let $r, s$ be integers such that $r \geq 0, s \geq 2$ and $r+s=k+1$. Then $G$ is generously $k$-fold transitive if and only if $\left(\chi^{\lambda}, \chi^{\mu}\right)_{G}=\delta_{\lambda \mu}$ for any characters $\chi^{\lambda}, \chi^{\mu}$ of $s_{n}$ such that dimension $\chi^{\lambda}=r$, dimension $\chi^{\mu}=s$ and $\chi^{\mu} \frac{1}{t}$ $\chi^{(n-s, s)}$.

A special case of this result was al so obtained by E. Bannai [1].

Notation. Let $r \leq n$; we write $\Omega^{(r)}, \Omega^{\{r\}}$ for the $S_{n}$-space of ordered, unordered $r$-point subsets of $\Omega$, respectively. We denote by $\pi^{(r)}, \pi^{\{r\}}$ the corresponding permutation characters. For the definitions and some properties of the irreducible characters of $S_{n}$ see [2], [4] or [8].

Received by the editors November 19, 1973.

AMS (MOS) subject classifications (1970). Primary $20 \mathrm{~B} 20$.

Copyright $\odot 1975$, American Mathematical Society 
If $H$ is a permutation group on a set $\Gamma$, we write $\operatorname{orb}(H, \Gamma)$ for the set of orbits of $H$ on $\Gamma$.

The next two lemmas are well known.

Lemma 1. Let $G$ act on the sets $\Gamma_{1}, \Gamma_{2}$, and let $\pi_{1}$ and $\pi_{2}$ be the corresponding permutation characters. Then

$$
\left(\pi_{1}, \pi_{2}\right)_{G}=\left|\operatorname{orb}\left(G, \Gamma_{1} \times \Gamma_{2}\right)\right|
$$

If $G$ is transitive on $\Gamma_{1}$ then $\left(\pi_{1}, \pi_{2}\right)_{G}=\left|\operatorname{orb}\left(G_{\alpha}, \Gamma_{2}\right)\right|$ for any point $a$ of $\Gamma_{1}$.

Lemma 2. Let $r \leq n$. Then

(a) $\pi^{\{r\}}=\Sigma_{i=0}^{r} \chi^{(n-i, i)}$

(b) $\pi^{(r)}=\Sigma_{\lambda} a_{\lambda} \chi^{\lambda}$ with $a_{\lambda}>0$, where the summation runs over all partitions $\lambda$ of $n$ of dimension less or equal to $r$;

(c) $\left(\pi^{(r)}, \chi^{(n-r, r)}\right)_{S_{n}}=1$.

Proof. (a) See, eg., $[8,4.2]$.

(b) [4].

(c) This follows easily from (a) and Lemma 1.

Proof of the Theorem. The group $G$ is $k$-fold transitive, and so by the Theorem [5, 14.3] mentioned at the beginning, $\left(\chi^{\lambda}, \chi^{\mu}\right)_{G}=\delta_{\lambda \mu}$ for characters $\chi^{\lambda}, \chi^{\mu}$ of $s_{n}$ with $\operatorname{dim} \chi^{\lambda}+\operatorname{dim} \chi^{\mu} \cdot \leq k$. Hence the character condition of the Theorem is by Lemma 2 equivalent to

$$
\left(\pi^{(r)}, \pi^{(s)}-\pi^{\{s\}}\right)_{G}=\left(\pi^{(r)}, \pi^{(s)}-\pi^{\{s\}}\right)_{S_{n}} .
$$

Now by Lemma $1,(1)$ is equivalent to

$$
\begin{aligned}
\operatorname{|orb}\left(s_{n}, \Omega^{(r)} \times \Omega^{(s)}\right)|-| \operatorname{orb}\left(G, \Omega^{(r)} \times \Omega^{(s)}\right) \mid \\
\quad=\left|\operatorname{orb}\left(s_{n}, \Omega^{(r)} \times \Omega^{\{s\}}\right)\right|-\left|\operatorname{orb}\left(G, \Omega^{(r)} \times \Omega^{\{s\}}\right)\right| .
\end{aligned}
$$

Let $\left(\left(\alpha_{1}, \cdots, \alpha_{r}\right),\left(\beta_{1}, \cdots, \beta_{s}\right)\right) \in \Omega^{(r)} \times \Omega^{(s)}$ and assume that $\left\{\alpha_{1}, \cdots\right.$, $\left.\alpha_{r}\right\} \cap\left\{\beta_{1}, \cdots, \beta_{s}\right\} \neq \varnothing$. Then $\left|\left\{\alpha_{1}, \cdots \alpha_{r}, \beta_{1}, \cdots, \beta_{s}\right\}\right| \leq k$. But $G$ is $k$-fold transitive, and so

$$
\left(\left(\alpha_{1}, \cdots, \alpha_{r}\right),\left(\beta_{1}, \cdots, \beta_{s}\right)\right)^{G}=\left(\left(\alpha_{1}, \cdots, \alpha_{r}\right),\left(\beta_{1}, \cdots, \beta_{s}\right)\right)^{s_{n}} .
$$

Hence 


$$
\begin{aligned}
\operatorname{|orb}\left(s_{n},\right. & \left.\Omega^{(r)} \times \Omega^{(s)}\right)|-| \operatorname{orb}\left(G, \Omega^{(r)} \times \Omega^{(s)}\right) \mid \\
& =\left|\operatorname{orb}\left(S_{n}, \Omega^{(r)} \dot{\times} \Omega^{(s)}\right)\right|-\left|\operatorname{orb}\left(G, \Omega^{(r)} \dot{\times} \Omega^{(s)}\right)\right|,
\end{aligned}
$$

where

$$
\begin{aligned}
\Omega^{(r)} \dot{\times} \Omega^{(s)}=\left\{\left(\left(\alpha_{1}, \cdots, \alpha_{r}\right),\right.\right. & \left.\left(\beta_{1}, \cdots, \beta_{s}\right)\right) \in \Omega^{(r)} \times \Omega^{(s)} \mid \\
& \left.\left\{\alpha_{1}, \cdots, \alpha_{r}\right\} \cap\left\{\beta_{1}, \cdots, \beta_{s}\right\}=\dot{x}\right\} .
\end{aligned}
$$

We obtain a similar expression also for the right-hand side of (2). Hence (2) is equivalent to

$$
\begin{aligned}
\mid \operatorname{orb}(G, & \left.\Omega^{(r)} \dot{\times} \Omega^{(s)}\right)|-| \operatorname{orb}\left(G, \Omega^{(r)} \dot{\times} \Omega^{\{s\}} \mid\right. \\
& =\left|\operatorname{orb}\left(s_{n}, \Omega^{(r)} \dot{\times} \Omega^{(s)}\right)\right|-\left|\operatorname{orb}\left(s_{n}, \Omega^{(r)} \dot{\times} \Omega^{\{s\}}\right)\right| .
\end{aligned}
$$

But $S_{n}$ is $(k+1)$-fold transitive, and so

$$
\left|\operatorname{orb}\left(s_{n}, \Omega^{(r)} \dot{\times} \Omega^{(s)}\right)\right|=1=\left|\operatorname{orb}\left(s_{n}, \Omega^{(r)} \dot{\times} \Omega^{\{s\}}\right)\right| .
$$

Thus (3) holds if and only if

$$
\left|\operatorname{orb}\left(G, \Omega^{(r)} \dot{\times} \Omega^{(s)}\right)\right|=\left|\operatorname{orb}\left(G, \Omega^{(r)} \dot{\times} \Omega^{\{s\}}\right)\right|,
$$

and (4) is equivalent to

$$
\begin{aligned}
& \operatorname{|orb}\left(G_{a_{1} \ldots a_{r}},\left(\Omega \backslash\left\{\alpha_{1}, \cdots, \alpha_{r}\right\}\right)^{(s)}\right) \mid \\
& =\left|\operatorname{orb}\left(G_{a_{1} \cdots a_{r}},\left(\Omega \backslash\left\{\alpha_{1}, \cdots, \alpha_{r}\right\}\right)^{\{s\}}\right)\right|
\end{aligned}
$$

for any $\alpha_{1}, \cdots, \alpha_{r} \in \Omega$.

Now (5) is equivalent by $[6,3.3]$ to saying that $G_{a_{1}} \ldots a_{r}$ is generously $s$-fold transitive on $\Omega \backslash\left\{a_{1}, \cdots, a_{r}\right\}$ for any $a_{1}, \cdots, a_{r} \in \Omega$, which in tum is equivalent to $G$ being generously $(r+s)$-fold transitive on $\Omega$ by $[6,3.1]$. The proof is now complete.

Remark 1. In the last section of [3] Frobenius obtained the character table of $M_{24^{\circ}}$. He remarks that the characters $\chi^{(21,1)}$ and $\chi^{(21,2,1)}$ of $S_{24}$ remain irreducible when restricted to $M_{24}$. The Theorem explains this remarkable fact, since $M_{24}$ is generously 5-fold transitive [6, 6.3].

Remark 2. A similar theorem for almost generous transitivity (as defined in [6]). can be found in [7].

Acknowledgement. The Theorem appears in my thesis [7]. I wish to thank my supervisor Dr. P. M. Neumann for his help, and the Science Research Council for their financial support. 


\section{REFERENCES}

1. E. Bannai, A note on characters of normal subgroup of multiply transitive permutation groups, J. Fac. Sci. Univ. Tokyo Sect. IA 20 (1973), 373-376.

2. A. J. Coleman, Induced repres entations with applications to $S_{n}$ and $G L(n)$, Queen's Papers in Pure and Appl. Math., no. 4, Queen's University, Kingston, Ont., 1966. MR 34 \# 2718.

3. G. Frobenius, $\ddot{U}$ ber die Charactere der mehrfach transitiven Gruppen, Berliner Berlichte, 1904, 558-571.

4. R. A. Liebler and M. R. Vitale, Ordering the partition characters of the symmetric group, J. Algebra 25 (1973), 487-489.

5. H. Nagao, Multiply transitive permutation groups, Lecture Notes, California Institute of Technology, Pasadena, 1967.

6. P. M. Neumann, Generosity and characters of multiply transitive permutation groups (submitted for publication).

7. J. Saxl, Multiply transitive permutation groups, Ph.D. Thesis, Oxford, 1973.

8. E. Snapper, Group characters and nonnegative integral matrices, J. Algebra 19 (1971), 520-535. MR $44 \# 1748$.

MATHEMATICAL INSTITUTE, 24-29 ST. GILES, OXFORD, ENGLAND

DEPARTMENT OF MATHEMATICS, UNIVERSITY OF ILLINOIS AT CHICAGO CIRCLE, CHICAGO, ILLINOIS 60680 